\title{
Contribución de la sociología en la formación docente. Un ejercicio de intervención en México
}

\author{
Contribution of sociology in training teacher. An intervention exercise in \\ Mexico
}

\section{Roxana Loubet-Orozco'}

\section{Resumen}

Este artículo presenta un estudio exploratorio y cualitativo que tuvo como objetivo identificar el tipo de reflexión de estudiantes de sociología acerca de la función social del fenómeno educativo, como resultado de la participación en un Seminario de Sociología de la Educación. Se realizó un análisis del contenido de los textos escritos producidos por los participantes durante el proceso de aprendizaje y las expresiones orales vertidas durante la interacción en el aula, teniendo como categorías centrales de reflexión, de tipo funcional o de tipo crítica. Los participantes fueron estudiantes de pregrado, de la licenciatura en Sociología, que al mismo tiempo, en su mayoría, eran docentes o tenían experiencia en esta actividad. En síntesis, se observó la importancia del estudio intencionado de la educación como fenómeno social para que se pueda percibir como tal; además, que la reflexión crítica emerge cuando el estudio y la comprensión de las aportaciones teóricas se conjuga con la práctica docente.

\section{Palabras clave}

Formación docente, sociología de la educación, reflexión crítica.

\section{Abstract}

This paper presents an exploratory and qualitative study aimed to identify the reflection of sociology students about the social function of the educational phenomenon as a result of participation in a Sociology of Education seminar. An analysis was made of the content of the written texts produced by participants during the learning process and the oral expressions expressed during the interaction in the classroom. The participants were students of the degree in Sociology, who at the same time, for the most part, were teachers or had experience in this activity. In summary, it was observed the importance of the intentional study of education as a social phenomenon so that it can be perceived as such; In addition, that critical reflection emerges when the study and understanding of the theoretical contributions is conjugated with the teaching practice.

\section{Keywords}

Teacher training, educational sociology, critical reflection.

1 Universidad Autónoma de Sinaloa; Facultad de Ciencias Sociales; Mazatlán, Sinaloa, México; roxloubet@uas.edu.mx 


\section{Introducción}

Con el objetivo de identificar el tipo de reflexión acerca del fenómeno educativo como resultado de la participación en un Seminario de Sociología de la Educación, se realizó una investigación cualitativa y exploratoria con un grupo de estudiantes de pregrado (o licenciatura) inscritos en esa carrera en la Universidad Autónoma de Sinaloa quienes, en su mayoría, laboraban o participaban en alguna institución escolar como profesores, o como apoyo docente y administrativo.

Uno de los antecedentes de esta inquietud fue el resultado de un proyecto de intervención realizado en la sierra oaxaqueña de México con profesores de educación básica de programas para niños migrantes (Loubet y Jacobo, 2013). En esta experiencia se observó que en la reflexión espontánea de los profesores participantes no había referencia alguna a la dimensión social de su práctica, ya que al principio, solo hicieron mención de problemas focalizados en el aula, como la indisciplina, el incumplimiento de tareas o problemas técnico-pedagógicos, mostrando con ello que el vivir y el desempeñarse en un contexto de marginación y pobreza no repercute automáticamente en el desarrollo de pensamientos críticos que pongan en duda el orden social o las relaciones de desigualdad. Fue hasta después de trabajar un taller de análisis de la educación de los niños migrantes como sistema, que se esbozaron algunas preocupaciones sobre, por ejemplo, las relaciones de género, la influencia del medio sociocultural, la desvinculación entre comunidades de origen y destino o la intencionalidad social del currículum.

Otra de las motivaciones para realizar la investigación que aquí se presenta, fue el hecho de que desde hace varias generaciones, en la carrera de Sociología de nuestra facultad se han inscrito profesores de los diversos niveles educativos, principalmente de nivel medio, con el objetivo inicial de posicionarse de mejor manera en sus centros de trabajo, dado que esta licenciatura es una de las opciones para perfilarse como docentes en 25 asignaturas de ese nivel (Historia, Formación cívica y ética, Introducción a las ciencias sociales, Filosofía, Estructura socioeconómica de México, Metodología de la investigación, entre otras, SEP, 2014). Tras unas primeras entrevistas con estudiantes y egresados acerca de la contribución de la carrera en su trabajo docente, se encontró que la mayoría dio cuenta de las capacidades para la investigación y el análisis, en tanto que solo quienes tenían formación en sociología de la educación hicieron, además, planteamientos críticos acerca de la función social de la educación y de su práctica. En ese sentido, se observó que tampoco basta con una formación en ciencias sociales para generar una reflexión social y crítica acerca de la práctica educativa.

A partir de lo anterior, y gracias al interés del grupo de sociología de la generación 2012-2016, se diseñó e implementó un Seminario de Sociología de la Educación como parte de las materias optativas que cursaron durante el semestre agosto-diciembre de 2015. El seminario sirvió como estrategia de intervención con el fin de promover en los estudiantes-docentes o futuros docentes una visión social y crítica del fenómeno educativo. Se partió del supuesto de que el seminario podría contribuir a focalizar la reflexión en este sentido y además, favorecer el desarrollo de un mayor compromiso con su desempeño profesional.

\section{Reflexión docente}

¿Qué se entiende por reflexión docente? Tomando como referencias básicas tanto a Dewey (1989) como a Schön (1992) se considera a la reflexión docente como un proceso que inicia con un estado de perplejidad ante situaciones determinadas de la práctica cotidiana del profesor, que encierra la capacidad de hacer visibles los elementos y relaciones que configuran una situación de enseñanza y aprendizaje con el apoyo de estrategias de investigación sistemática y objetiva. Por otro lado, que la reflexión docente im- 
plica también la reconsideración del carácter social de la educación y del rol como parte de un contexto sociocultural, político y económico (Durkheim, 1975; Van Manen, 1977; Valli, 1990; Pérez Gómez, 1993; Kemmis, 2007); pero, este tipo de reflexión demanda herramientas conceptuales que permitan develar lo que se presenta en la apariencia de las relaciones educativas. De ahí que, mediante el Seminario, se propuso provocar la reflexión social y crítica de los estudiantes-docentes.

Inspirados en la teoría del conocimiento de Habermas², tanto Van Manen (1977) como Kemmis (2007) coinciden al proponer tres niveles de reflexión docente: una técnica, una práctica, y otra crítica. La reflexión técnica ligada a una visión lineal positivista y un enfoque conductista se concentra en la aplicación eficiente de las estrategias y el cumplimiento de los objetivos propuestos en el currículum; la reflexión práctica, con una perspectiva interpretativa, reconoce una realidad construida en la interacción social en el marco de contextos culturales específicos de la cotidianeidad, una construcción interpretativa de las experiencias que puede poner en cuestionamiento los medios y los objetivos de la enseñanza; por último, la reflexión crítica, cuyo sentido es eminentemente social (ético y sociológica) y que cuestiona las posibilidades y consecuencias de las acciones educativas con una clara intención de cambio social y emancipación.

Baildón y Sim (2009) conceptualizan el pensamiento crítico como una forma de práctica social situada histórica, cultural, económica y políticamente. En el sentido de Kemmis (2007), reflexionar críticamente es analizar la arquitectura de las estructuras de pensamiento y de las prácticas escolares que son perjudiciales. La reflexión crítica advierte los componentes sociopolíticos que subyacen en relación al sistema social en el que se inscribe el currículum y la práctica del maestro, dando cuenta del currrículum oculto.

Con un enfoque cercano, Valli (1990) propone tres tipos de reflexión moral en el docente: 1) deliberativa; 2) relacional y 3) crítica. La deliberativa expresa el comportamiento ético del profesor en función de su relación con los estudiantes, con la escuela y las políticas educativas. La relacional se distingue en que el profesor es un «cuidador», reflexiona sobre la realidad de los estudiantes para orientarlos a una vida digna y de respeto, más que en los resultados de los exámenes. Por último, en la reflexión crítica, se parte de que la escuela es una institución que contribuye a reproducir las relaciones de clase, de género, de raza, por lo que los profesores deben contribuir para cambiar las estructuras de la escuela y las prácticas de enseñanza que refuerzan esas relaciones; deben promover que sus estudiantes tomen conciencia de esto y desarrollen capacidades de emancipación. Desde esta última perspectiva las escuelas deben ser lugares de empoderamiento y transformación social.

En el mismo sentido de Valli (1990), y además de la reflexión técnica y práctica, Jacobo (1997) propone una dimensión social, dividida en funcional y en crítica. En la reflexión socio-funcional el profesor se preocupa por mejorar su trabajo y ofrecer un servicio de calidad, sin cuestionar los objetivos educativos o las formas y contenidos propuestos por el sistema educativo o la institución escolar; asume que su papel es lograr los aprendizajes de los contenidos y valores prescritos por la sociedad para propiciar la integración social de sus estudiantes. En tanto que la reflexión socio-crítica convoca el cambio social, a la formación de educandos con capacidad de romper con las estructuras sociales de desigualdad e inauguración de otras más justas.

2 En su obra Conocimiento e interés, Habermas (1995) aborda tres tipos de intereses cognitivos: (1) técnico, inscrito en el marco del paradigma positivista y correspondiente a las ciencias empírico analíticas; (2) práctico, asentado en un paradigma fenomenológico y en las ciencias histórico hermenéuticas y (3) crítico, basado en las teorías críticas y en una perspectiva emancipadora. 
La búsqueda de literatura para ampliar nuestro conocimiento en la cuestión arrojó la identificación de escasas investigaciones orientadas al conocimiento del pensamiento social docente o creencias educativas desde la dimensión de lo social; entre ellas, destacamos el estudio de Fernandes dos Santos (2008) en Portugal; los de Pérez Sánchez (2000), Montané, Aneas y Olivé (2009) en España; de Gómez y Niño (2015) en Colombia; Catalán (2004), Jiménez y Fardella (2015) en Chile; y en México, los de Macotela, Flores y Seda (2001), Aguilar Nery (2003) y el de Tapia y Valenzuela (2015).

De acuerdo con los resultados de algunos de los trabajos revisados, los profesores tienden a concebir a la educación y al aprendizaje como un proceso de carácter y beneficio individual, asociando, por ejemplo, el éxito escolar a las capacidades y el nivel de motivación de los alumnos (Montané, Aneas y Olivé, 2009; Pérez Sánchez, 2000), o que la escuela es importante porque ofrece formación para la vida adulta y se debe enseñar lo que beneficie al individuo (Macotela, Flores y Seda, 2001; Aguilar Nery, 2003).

Por otro lado, en Fernandes dos Santos (2008) los profesores concebían su actividad desde una perspectiva ética, asumiendo la responsabilidad de formar e integrar a las nuevas generaciones para renovar el tejido social, conjugando el respeto por la tradición con la preparación hacia el futuro, aunada a una visión crítica en torno a las condiciones de la profesión, el perfil del colectivo docente y a la posición condescendiente del gobierno. En Bogotá, Gómez y Niño (2015) indagaron acerca el posicionamiento del rol de 20 profesores de secundaria, mostrando que la mayoría se percibía como formador y orientador de los estudiantes para la vida social; lo interesante de este estudio, es que al comparar profesores del área de ciencias sociales con los del área de matemáticas, ninguno de los primeros se consideró ligado a un modelo instruccional -como «el que enseña» o el ser «un modelo a seguir»- en tanto que el 90\% de los segundos sí se identificó dentro de dicha categoría. En Santiago de Chile, en el marco de educación de inmigrantes, Jiménez y Fardella (2015) encontraron que los profesores de educación básica pensaban la función de la escuela como «espacio de adaptación cultural», aunque reconocieron discursos con la idea de la escuela como «espacio de aprendizaje intercultural».

En México, Aguilar Nery (2003) cuestionó a profesores-estudiantes de una institución formadora de docentes en la ciudad de Tijuana y encontró, que la mayoría veía a la escuela como formadora de trabajadores y ciudadanos, así como instancia de progreso y movilidad social; y supusieron que la sociedad esperaba que fueran excelentes transmisores de conocimientos, valores y actitudes, aunque el $44 \%$ de la muestra se consideró ser un sujeto crítico y reflexivo. Tapia y Valenzuela (2015) observaron en escuelas de la periferia de Nogales que profesores de secundaria perciben el problema del alumnado inmigrante desde una perspectiva individualista y adaptativa, al expresar, por ejemplo, uno de ellos: «Vendría siendo el maestro el pilar fundamental para darle la fortaleza a ese alumno y que pueda sentirse tan seguro de su persona como alumno, como estudiante y como esa persona que debe de buscar el salir adelante con sus problemas o sin sus problemas» (cit. en Tapia y Valenzuela, 2015:80).

Otro estudio reciente en México, es el de Armenta, Ibarra y Jacobo (2015), el cual tuvo como propósito describir las transiciones de educadores en contextos adversos para superar el conflicto emocional, y si bien no se orientó específicamente a indagar la dimensión social, sí se observó que, a partir de dos talleres - uno para escribir cartas y otro de reflexión profesional- solo 3 de 9 profesores de la escuela primaria de la penitenciaría Islas Marías experimentó una reflexión de tipo socio-funcional y ninguno de ellos, una reflexión socio-crítica. 
En síntesis, se observa que los profesores tienden a pensar a la educación y a sí mismos más desde una perspectiva individualista y funcional, y que, a pesar de identificar condiciones de desigualdad, control del Estado o dominio cultural, la reflexión social crítica de parte de estos colectivos es incipiente. De ahí que formulamos como supuesto de partida que para construir un pensamiento crítico de la educación como un fenómeno social es necesario un estudio y análisis intencionado que rompa con las perspectivas «románticas» de la institución escolar, cuya visión conservadora y positivista le infiere un carácter aséptico con el «único» noble propósito de potenciar el desarrollo de los individuos, invisibilizando su función reproductora (Bourdieu y Passeron, 1996). En esta misma línea, Krichesky, et al., (2011) proponen la necesidad de programas de formación docente que brinden espacios de discusión acerca del rol de la escuela y la función de los profesores frente a la sociedad en aras de combatir la «neutralidad académica» que fortalece la normalización del estatus dominante.

La investigación sobre práctica y formación inicial del docente ha mostrado que los futuros maestros, novatos o inclusive expertos, llevan consigo la carga de experiencias con las que transitaron por su vida escolar (Pérez Gómez, 1993), que asumen maneras de hacery de pensar-parafraseando a Durkheim-adquiridas en su paso por la escuela desde la infancia; por otro lado, como sujetos inmersos en contextos sociales específicos y de posiciones en las relaciones de poder, también han construido un esquema de creencias como marco interpretativo del mundo.

Dado lo anterior, emergieron las siguientes inquietudes: ¿Cómo puede contribuir la sociología con un pensamiento crítico y alternativo para educar para la justicia social? ¿El estudio del fenómeno educativo desde la sociología puede generar espacios y momentos de reflexión crítica por parte del docente acerca de su práctica educativa? ¿Cómo puede contribuir el conocimiento de las aportaciones sociológicas en relación a las instituciones e interacciones educativas en la reflexión docente?

\section{Pertinencia de la sociología en la formación docente}

En la perspectiva de Florestán Fernández (cit. en Pereyra y Pontremolli, 2014) la inclusión del estudio de sociología en los programas educativos puede contribuir a formar una conciencia cívica en los ciudadanos, promoviendo una ética de responsabilidad y una actitud de autonomía crítica. Para Pereyra y Pontremoli (2014) la sociología debería despertar la imaginación sociológica crítica y comprometida de los educandos, ayudando a identificar la brecha entre la realidad social percibida y el pensamiento utópico; por lo cual, la sociología podría recordarles permanentemente a los estudiantes que el mundo puede ser diferente, de ahí que propongan que debería ser una materia curricular que permitiera a todos los estudiantes tener un espacio de reflexión en torno a temáticas de la sociedad, sus problemas y desafíos, ya que, reflexionan: «Si el interés pedagógico se centra más en el uso de las tecnologías que en las interacciones sociales esa oportunidad se habrá perdido y confirmaría la hipótesis, sostenida por varias autoridades educativas, que la sociología está pasada de moda» (Pereyra y Pontremoli, 2014: 157).

La importancia de la sociología y específicamente el valor de la sociología de la educación en la formación docente es una cuestión añeja; análisis e investigaciones al respecto se encuentran ya en Reavis (1928) y en Corbally (1942) en Estados Unidos, McNamara (1972) en Gran Bretaña, y en Daine y Foster (1976) en Canadá. Sin embargo, su presencia en tiempos actuales es difusa y tiende a desaparecer en los planes de estudio de formación inicial ${ }^{3}$ e incluso en la investigación de los departamentos de sociología (DeCésare, 2003).

3 Por ejemplo, en México, el programa de Licenciatura en educación primaria, 2012, incluye ciertos contenidos que se vinculan con lo social en la materia de Filosofía, pero no se incluye la asignatura de Sociología de la educación. 
Guerrero Serón (2007) argumenta que la sociología de la educación dota al docente de los instrumentos teóricos y analíticos para comprender y actuar sobre el contexto social donde se está formando y donde va a desarrollar su trabajo; se trata, entonces de sacar a la luz las creencias irracionales ocultas en la memoria colectiva, y en ese sentido, mostrar que las cosas no son siempre lo que parecen. En este mismo orden de ideas, Sáenz (2009) coincide en que la sociología puede contribuir a liberarse de las determinaciones sociales incrustadas en el alumnado de magisterio a lo largo de su vida escolar, a romper el habitus encarnado en los procesos de estructuración social; en tanto que para García Pérez (2006), la perspectiva crítica se revela como una apoyatura fundamental para generar conciencia en los docentes de las implicaciones sociales de su actos profesionales.

Ciertamente, la educación es un medio para desenvolverse de manera más inteligente en el mundo; la escuela, como un microcosmos de la sociedad, permite socializar el conocimiento acumulado en un entorno de interacción, y en la cual, desde la visión de la pedagogía crítica, es posible también constituir espacios de autonomía y resistencia para generar nuevas miradas del mundo (Giroux, 2004), orientar acciones pedagógicas y un pensamiento crítico para educar con sentido de justicia social, entendiendo por ello no solo redistribución de bienes materiales, sino igualdad de oportunidades, de acceso al poder, al conocimiento y al reconocimiento de las diferencias (Murillo y Hernández, 2011). No obstante, para que los docentes construyan este tipo de pensamiento se requiere, en primer lugar, que reconozcan las estructuras de dominación en las que se rige un sistema educativo, pues, como inquieren Méndez y Murillo (2017:394) «نes posible llevar a cabo una labor contra hegemónica careciendo de una formación crítica del profesorado?». Si se quiere incidir en el desarrollo de una sociedad más justa, es preciso identificar las bases en las que está construida la desigualdad y, en ese sentido, el cómo la escuela se coadyuva en esta situación.

La reflexión crítica acerca de la función social de la educación, de la escuela y del profesorado, posibilitará asumir conscientemente la práctica docente y dar sentido comprometido a los planteamientos de educación para la justicia social, desarrollo sustentable, perspectiva de género y derechos humanos. Sabemos que no es garantía, pero al situar socialmente el fenómeno educativo desde un marco teórico crítico se puede contribuir en la construcción de un conocimiento que empodere las acciones educativas demandadas por nuestras sociedades para salir del subdesarrollo y enfrentar la lógica del capitalismo.

Desde esta perspectiva, es necesario estudiar las relaciones de dominación y reproducción que se presentan en la escuela para poder proponer condiciones alternativas. En tanto no se tenga conciencia de la necesidad de emancipación se seguirá legitimando una manera de hacer y de pensar.

\section{Método}

La investigación se realizó en la ciudad de Mazatlán, Sinaloa, al noroeste de la República Mexicana, con alcance exploratorio y enfoque cualitativo, teniendo como objetivo identificar el tipo de reflexión de estudiantes de sociología acerca del fenómeno educativo como resultado de la participación en un Seminario de Sociología de la Educación, programado como materia optativa en el semestre agosto diciembre de 2015. Específicamente, se pretendió observar si el participar en el Seminario promovía en el pensamiento de los estudiantes una reflexión de tipo social y crítica acerca de la educación.

El propósito del Seminario de Sociología de la Educación fue contribuir en la construcción de una reflexión que situara social y críticamente al fenómeno educativo, mediante el estudio intencionado de dicho fenómeno a través de las teorías sociológicas, posicionando su institucionalización como espacio de pro- 
ducción y reproducción de prácticas sociales e instancia de constitución de sujetos sociales, para desde ahí analizar el sistema educativo mexicano actual y su relación con distintos ámbitos del orden social. Esto, debido a que en observaciones previas se encontró que, en general, los estudiantes de sociología no alcanzaban a visualizar con precisión el proceso educativo como objeto de interés para su profesión, y manifestaban opiniones ambiguas a partir de sus experiencias personales o centradas en perspectivas psicológicas y pedagógicas.

Se desarrolló en cuatro ejes temáticos: La educación como objeto de la sociología; el carácter social de la educación, la escuela y el docente; la institución educativa en el contexto actual; y tópicos de la educación en México para el debate.

La dinámica que se propuso fue de tipo investigativo (Pérez Puente, 2010), es decir, de estudio, reflexión y producción de ideas. Se partió de la premisa de que un seminario es un encuentro entre iguales que interactúan guiados por un interés intelectual para la construcción de un saber; una estrategia de formación que implica la participación activa de todos los participantes en tanto que cada seminarista aporta su estudio, experiencia e interpretación a la tarea común y todos se benefician de la acción colectiva. Asimismo, que el aprendizaje se genera a partir de la actividad reflexiva, y ésta se nutre de la información que resulta de la investigación y la discusión compartida. En ese sentido, se tuvo como base el diálogo y las relaciones dialógicas, en un ambiente de horizontalidad y respeto ante los integrantes y ante el conocimiento mismo, de escucha activa ante las ideas expresadas por el resto y de responsabilidad al asumir las tareas.

Por otro lado, la estrategia pedagógica se basó en una adaptación del modelo propuesto por Jackson (1989) de tres fases de interacción: preactiva, interactiva y postactiva. La fase preactiva consistió en el primer momento del seminario donde los participantes compartieron sus ideas acerca de aspectos del fenómeno educativo, antes del estudio de la bibliografía propuesta por eje temático. Para la fase interactiva se participó con base en la lectura especializada: se estudiaban previamente los temas, se exponían las ideas e inquietudes durante las sesiones de clase generando la conversación y discusión grupal. Finalmente, la fase postactiva fue un momento de reflexión posterior al estudio y sesiones de clase, en el cual se trató de que los participantes se autoevaluaran, haciendo una comparación de su pensamiento entre el antes y después del estudio intencionado.

\section{Población y muestra}

El grupo estuvo constituido por 7 mujeres y 7 hombres, con una media de edad de 34 años (33 las mujeres y 34 los hombres), en un rango de edad de 22 a 52 años; 8 (57\%) tenía actividad laboral en ese momento, 9 (64\%) contaban con alguna experiencia en actividades docentes, 8 (57\%) tenían otros estudios ( 2 licenciados en educación primaria y 1 en psicología; 2 técnicos en humanidades y 3 técnicos en áreas no afines), 5 (36\%) habían tomado cursos sobre el tema de educación o de pedagogía y solo $2(14 \%)$ dijeron tener lecturas en sociología de la educación.

Los participantes se clasificaron de acuerdo a la antigüedad en actividades docentes en expertos (más de 5 años), principiantes (menos de 5 años) (Marcelo, 2008) y sin experiencia (Tabla I). 
Tabla I. Perfil de la muestra

\begin{tabular}{|c|c|c|c|c|c|c|}
\hline $\mathbf{P}$ & SEXO & EDAD & $\begin{array}{l}\text { ESTUDIOS } \\
\text { PREVIOS }\end{array}$ & $\begin{array}{c}\text { TIPO DE ACTIVIDAD } \\
\text { DOCENTE }\end{array}$ & $\begin{array}{l}\text { ANTIGÜEDAD } \\
\text { DOCENTE }\end{array}$ & $\begin{array}{c}\text { NIVEL } \\
\text { DE EXPERIENCIA }\end{array}$ \\
\hline 1 & M & 52 & Lic. Psicología & Primaria/Supervisión & 34 años & Experta \\
\hline 2 & M & 43 & Bachillerato & Servicio social & 6 meses & Principiante \\
\hline 3 & $M$ & 33 & Lic. Educ. Primaria & Primaria & 8 años & Experta \\
\hline 4 & $M$ & 22 & Bachillerato & No & - & Sin experiencia \\
\hline 5 & $M$ & 28 & Secretariado & No & - & Sin experiencia \\
\hline 6 & M & 22 & Bachillerato & No & - & Sin experiencia \\
\hline 7 & M & 33 & Bachillerato & Servicio social & 6 meses & Principiante \\
\hline 8 & $\mathrm{H}$ & 45 & Oficial de armas & Instructor/docente inglés & + de 10 años & Experto \\
\hline 9 & $\mathrm{H}$ & 28 & Lic. Educ. Primaria & Primaria & 5 años & Experto \\
\hline 10 & $H$ & 39 & Bachillerato & No & - & Sin experiencia \\
\hline 11 & $\mathrm{H}$ & 38 & Inst. eléctricas & Asistente en secundaria & 2 años & Principiante \\
\hline 12 & $\mathrm{H}$ & 40 & Artes plásticas & Tallerista/telesecundaria & 25 años & Experto \\
\hline 13 & $H$ & 26 & Bachillerato & Programa migrantes & 2 años & Principiante \\
\hline 14 & $H$ & 26 & Música & No & - & Sin experiencia \\
\hline
\end{tabular}

$\mathrm{P}=$ Participante

Fuente: elaboración propia.

\section{Recogida y análisis de la información}

Como parte del proceso del Seminario se aplicaron instrumentos y estrategias de recolección de datos, mismos que fueron utilizados para la investigación:

- Cuestionario inicial de 10 preguntas para determinar el perfil de los participantes y explorar su experiencia en relación al ámbito educativo, como docentes y como estudiantes.

- Textos escritos (preactivos) producidos por los participantes antes del estudio de cada eje temático, con base en una guía de preguntas formuladas para garantizar que se expresaran las ideas acerca de lo propuesto para el estudio.

- Registro de conversaciones, exposiciones y discusiones en el aula mediante una bitácora elaborada en el momento posterior a cada sesión, apoyada con recursos de grabación de video.

- Textos escritos (postactivos) producidos al término del Seminario. Si bien se esperaba que estos textos se construyeran inmediatamente después de cada eje temático, las condiciones laborales y dedicación al Seminario impidieron que fuera así; por lo que solo se hizo un texto postactivo al final, considerando la autoevaluación de todos los temas. Por otro lado, no todos pudieron establecer con claridad las diferencias de su pensamiento, por lo que se deduce la necesidad de orientar con mayor cuidado y precisión el proceso de instrucción para su elaboración.

A partir de la observación de los documentos producidos durante el Seminario y de la interacción cara a cara emergieron cuatro dimensiones para el análisis:

1. Actividad y experiencia docente. Se distinguió a los participantes de acuerdo al tipo y tiempo de experiencia como docentes o actividades de apoyo: Expertos, Principiantes y Sin experiencia.

2. Nivel de estudio. Se evaluó a partir de las exposiciones y participación de cada estudiante durante el Seminario. 
3. Nivel de comprensión de las teorias y análisis sociológicos. Al igual que el nivel de estudio, se evaluó con las exposiciones individuales y participación en aula, además del análisis de los textos postactivos. Como criterio de medición se consideró el hecho de que solo transcribieran o citaran los documentos leídos sin mayor interpretación o reflexión, y la proporción de ambigüedades plasmadas en la expresión de sus ideas.

4. Tipo de reflexión en torno a los temas abordados. Para tipificar la reflexión en su dimensión social se tomó en cuenta lo siguiente:

a. Concepción del proceso educativo como fenómeno social o por el contrario, de interés individual.

b. Concepción de la educación en el sentido socio-funcional, considerando como indicador la idea rectora de integración social sin cuestionamiento del orden o desvinculada del sentido histórico y político.

c. Concepción socio-crítica tomando como indicador la idea rectora de la educación como reproductora de un orden dominante y situada en un marco de relaciones históricas y políticas.

Las valoraciones se hicieron en función del sentido predominante en la expresión de las ideas en aula y principalmente en sus textos postactivos.

\section{La función del fenómeno educativo en la reflexión de los estudiantes de sociología}

Desde la tradición clásica, se espera que el sociólogo reflexione en relación a la función de los fenómenos sociales, tanto en el sentido funcional de su contribución al orden, como en la mirada crítica para poner al descubierto las relaciones subyacentes de dominación. De esta manera, se considera básico que un profesional de la sociología interesado en el tema de la educación, se detenga a pensar en la función social de esta problemática; que la entienda como un fenómeno que se constituye en relación a contextos sociales históricos y específicos, instituyendo en la conciencia de los individuos el ser social, en procesos de socialización. Que tiene funciones prescriptivas y coercitivas, pues a través de estos procesos se interiorizan y se imponen las normas, los valores y los roles que rigen la convivencia, construidos colectivamente para su propia renovación.

\section{a. Concepciones iniciales en torno a la función educativa ${ }^{4}$}

Los resultados del análisis muestran que la mayoría de los estudiantes de esta investigación, con alguna experiencia docente y sin ella, no tenían conceptualizado el fenómeno educativo desde la perspectiva sociológica, pues sus ideas iniciales fueron expresiones de sentido común limitando la función de la educación y de la institución escolar más orientada al beneficio individual, observándose dos categorías: formar para la vida y la profesión y la adquisición de conocimiento. En el mismo tenor, en los planteamientos sobre el docente se esbozó una visión ligada a la asimilación de conocimientos individuales, o en los que no se explicita su función social; se le percibió más como guía, mediador, promotor y con vocación (Tabla II).

4 En este apartado solo se analizan las reflexiones en torno al primer eje temático: carácter social de la educación. 


\section{Tabla II. Ideas iniciales acerca de la función de la educación y del docente}

Nivel de experiencia Frases

\begin{tabular}{ll}
\hline & Función de la educación \\
\hline Experta & «Formar para la superación personal» (P3). \\
\hline Experto & «La educación tiene la función de formar individuos integrados a los diferentes saberes de la vida, para poder desarrollar los conocimientos en el campo profesional» (P9). \\
\hline Experto & «Capacitar a los miembros de una sociedad en sus diferentes niveles de educación» (P8). \\
\hline Experto & «Proceso de capacitación y asimilación de conocimientos organizados» (P12). \\
\hline Experto & «Contribuir a los proyectos de felicidad del sujeto» (P9). \\
\hline Principiante & "Formar y generar conocimiento en los individuos, no solo en lo académico, sino también en lo cultural y lo social» (P2). \\
\hline Sin experiencia & «Proporcionar herramientas de conocimiento lo mejor posible para formarlos y prepararlos en una sociedad» (P10). \\
\hline Experto & Función del docente \\
\hline Principiante & «Mediador entre el alumno y el conocimiento» (P9). \\
\hline Sin experiencia & «Gúa rumbo al conocimiento» (P2). \\
\hline Sin experiencia & «Estimular conocimientos, actitudes y valores» (P4). \\
\hline
\end{tabular}

Fuente: elaboración propia.

Por otro lado, se identificaron algunos textos donde se observa el fenómeno educativo desde una perspectiva social, aunque funcional, como en lo observado por Aguilar Nery (2003), Fernandes dos Santos (2008) y Gómez y Niño (2015), concibiendo a la educación, a la escuela y al docente en el sentido integrador (Tabla III).

Tabla III. Dimensión social en ideas iniciales

Nivel de experiencia Frases

\begin{tabular}{|c|c|}
\hline & Función de la educación \\
\hline Experto & «Para funcionar en la sociedad; para integrarse en tareas específicas de servicio a los demás» (P8). \\
\hline Experto & «Formar ciudadanos que desarrollen sus competencias y contribuyan en los procesos sociales y profesionales» (P9) \\
\hline \multirow[t]{2}{*}{ Experto } & «Producir movilidad social» (PI). \\
\hline & Función del docente \\
\hline Experto & «Guiar a los futuros ciudadanos para tener más valores para mejorar la sociedad» (P9). \\
\hline Experto & «Compromiso social de mediador y guía para acompañar a los alumnos» (PI). \\
\hline Experto & «Promotor que con su práctica reoriente las acciones y los comportamientos, tanto personales como de sus alumnos» (PI). \\
\hline Principiante & «Educarnos, formar ciudadanos» (P2). \\
\hline Sin experiencia & «Formar a los individuos reflexivos, analistas, para contribuir a un mejor desarrollo productivo, político y social, y civilizarlos con orden» (P10). \\
\hline
\end{tabular}

Fuente: elaboración propia.

Estas expresiones citadas con carácter social fueron producidas por tres de los participantes expertos, un principiante y uno sin experiencia. Pero, en general, en la reflexión de estos integrantes también se encontró una visión individualista, con un incipiente sentido crítico en relación a la función social, como se observa en las tablas 1,2 y 3.

Las referencias críticas se dieron en relación a las condiciones actuales del sistema educativo mexicano ${ }^{5}$, principalmente ligadas a las políticas educativas, la corrupción en el sistema de contratación de personal, poco compromiso de docentes, autoridades y familia, mala formación docente o desigualdad económica (Tabla IV).

5 Estas expresiones se dieron como respuesta a una de las preguntas en el cuestionario inicial para determinar el perfil: Desde su perspectiva ¿qué problemas observa en el sistema educativo mexicano? Como estudiante, como padre de familia o como ciudadano. 


\section{Tabla IV. Ideas iniciales con sentido crítico}

\begin{tabular}{|c|c|}
\hline Nivel de experiencia & Frases \\
\hline Experto & $\begin{array}{l}\text { «El problema principal de nuestro sistema educativo es la forma en la que está estructurado en función de políticas poco aplicables que tienden a la imitación de otros } \\
\text { modelos» (P1). }\end{array}$ \\
\hline Principiante & «Ya no existen maestros con vocación y si hay, son pocos... los alumnos y padres de familia ya no le dan la importancia a la educación... »(P11). \\
\hline Experto & «Uno de los problemas de la educación es el poco compromiso de alumnos, de docentes y padres de familia» (P9). \\
\hline Experto & «En ocasiones, el personal no está capacitado para estar frente a grupo... los compadrazgos... no se respetan los lineamientos» (P3). \\
\hline Sin experiencia & «El método de la educación no funciona para todos» (P10). \\
\hline Experto & «La formación integral del sujeto en un ser más crítico y reflexivo para transformar su entorno en todos los contextos; para resolver situaciones o problemas de su entorno» (P1). \\
\hline Principiante & «La educación es muy limitada, no todos tienen acceso a ella por cuestiones de economía» (P11). \\
\hline
\end{tabular}

Fuente: elaboración propia.

No obstante, la mayoría de estas ideas manifiestan un malestar derivado más de la experiencia como profesores, padres de familia o estudiantes que como estudiosos de la sociología; expresiones que, si bien muestran sensibilidad, no demuestran una reflexión teórica ni enfocan el problema como un fenómeno social más allá del sentido común.

\section{b. Concepciones postactivas acerca del fenómeno educativo}

Posterior al estudio y a la interacción en el aula, en las conversaciones y en el análisis de textos fue posible distinguir elementos que indicaron un cambio de posición frente a la educación y sobre todo, a la educación institucionalizada. Pasó de percibirse como un fenómeno neutral e individualizante, a uno construido socialmente, provisto de ideología y de mecanismos de control social.

En los textos postactivos dieron cuenta de la visión que tenían antes del Seminario y cómo, después de él, había cambiado. En general, las ideas se perfilaron en el sentido de que si bien, la educación provee saberes y contribuye en la formación de los individuos, ésta tiene un carácter social; que los centros escolares y la acción docente se orienta a partir de las necesidades mismas de la sociedad, además de proyectarse en ella la cultura y las relaciones de poder. En ese sentido, ser más conscientes de su participación.

En el análisis de sus textos reflexivos se encontraron ideas críticas de la política global y el enfoque por competencias, legitimación del Estado, programas educativos al servicio del capital y del mercado, formación para la estratificación social, discriminación y desigualdad relacionadas con el capital cultural y económico, currículum oculto, violencia simbólica, y principalmente, estructuración a partir de la ideología de las clases dominantes (ver Tabla IV). 


\title{
Tabla V. Ideas postactivas con sentido crítico
}

\author{
Nivel de experiencia Frases

Experto
responder a las tendencias y exigencias de la globalización» (P3).

Experto $\quad$ «Vemos un sistema centrado en un modelo por competencias que busca preparar a sujetos para el trabajo... observando que la educación está al servicio del capital» (P12).

\begin{tabular}{|c|c|}
\hline Experto & $\begin{array}{l}\text { «Las escuelas funcionan en beneficio de la cultura dominante para reproducir la lógica y los valores de la sociedad existente; (...) las escuelas utilizan sus recursos materiales } \\
\text { e ideológicos para reproducir las relaciones sociales y las actitudes y habilidades necesarias para ocupar lugares específicos de las clases en las divisiones sociales de } \\
\text { trabajo»(PI). }\end{array}$ \\
\hline Experto & $\begin{array}{l}\text { «El modelo por competencias proviene de una concepción de 'capacidad'. Los más competentes o bien más capaces, serán los más idóneos para realizar las labores que la } \\
\text { sociedad y que la producción laboral demanden. Entonces ¿no es el modelo por competencias un modelo excluyente?» (P9). }\end{array}$ \\
\hline Experto & $\begin{array}{l}\text { «La clase social, género o etnia explica buena parte de la actitud del alumnado. El currículum oculto afecta bastante a los estudiantes. Las escuelas con alumnos de clase so- } \\
\text { cial obrera les enseñan a obedecer, mientras que las escuelas con alumnos de clase ejecutiva, o empresarial, o política, les enseñan a pensar, a ser creativos e independientes. } \\
\text { Lo que se castiga en un tipo escuela por el alumnado que tiene se premia en la otra, en la que tiene otro tipo de alumnado» (P8). }\end{array}$ \\
\hline Principiante & $\begin{array}{l}\text { «La escuela no se puede analizar separadamente del contexto económico y social... la escuela como un lugar político, por ende, habrá en ella enfoques políticos y normativos, } \\
\text { es decir, habrá ideología» (P2). }\end{array}$ \\
\hline Principiante & $\begin{array}{l}\text { «En el aula, los docentes y alumnos generan discriminación; (...) se manifiestan formas de violencia simbólica ya sea aislando o rechazando al alumno ... discriminándolo } \\
\text { verbal o físicamente... el docente no pone atención a estas situaciones» (P11). }\end{array}$ \\
\hline
\end{tabular}

Sin experiencia «El docente es el agente que reproduce el orden social establecido en el cual está inmerso y su función es que el alumno asimile esos saberes y esa moral cívica, colectiva que necesita la sociedad, e irse independizando de la socialización de la familia para interactuar en la sociedad» (P10).

Fuente: elaboración propia.

En general, a partir de estos resultados se puede inferir que efectivamente el estudio de la sociología de la educación puede contribuir a que los profesores en activo rompan con sus esquemas individualistas de la educación y adopten una mirada crítica, tal como lo proponen Sáenz (2009), Guerrero Serón (2007) y García Pérez (2006).

\section{Reflexión crítica, experticia, dedicación y comprensión teórica}

Si bien se pueden observar resultados positivos en el cambio de concepción dado que poco más de la mitad de los participantes en el Seminario vertieron ideas categorizadas aquí como críticas, también es notorio que esto no se generó con fuerza suficiente en todos ellos, observándose como posibles factores relacionados dedicación al estudio, comprensión teórica y nivel de experiencia docente (Tabla VI).

Por un lado, quienes proyectaron expresiones más críticas son al mismo tiempo expertos, con alto nivel de dedicación y comprensión. Por otro, quienes tuvieron nivel medio de expresión crítica y con menor nivel de experiencia docente, coincidieron en dedicación y comprensión de alta a media, en tanto que en los expertos fue de media a baja. 
Tabla VI. Nivel de reflexión crítica por participante

\begin{tabular}{|c|c|c|c|c|c|}
\hline $\mathbf{P}$ & SEXO & $\begin{array}{l}\text { REFLEXIÓN } \\
\text { CRÍTICA }\end{array}$ & $\begin{array}{c}\text { NIVEL } \\
\text { DE EXPERIENCIA }\end{array}$ & $\begin{array}{c}\text { NIVEL } \\
\text { DE ESTUDIO }\end{array}$ & $\begin{array}{c}\text { NIVEL } \\
\text { DE COMPRENSIÓN }\end{array}$ \\
\hline $\mathrm{Pl}$ & M & Alta & Experta & Alto & Alto \\
\hline P8 & H & Alta & Experto & Alto & Alto \\
\hline P9 & H & Alta & Experto & Alto & Alto \\
\hline P3 & M & Media & Experta & Medio & Bajo \\
\hline P12 & H & Media & Experto & Bajo & Medio \\
\hline P2 & M & Media & Principiante & Alto & Alto \\
\hline P11 & H & Media & Principiante & Medio & Medio \\
\hline P10 & H & Media & Sin experiencia & Alto & Medio \\
\hline P7 & M & Baja & Principiante & Bajo & Bajo \\
\hline $\mathrm{P} 13$ & H & Baja & Principiante & Bajo & Bajo \\
\hline P4 & M & Baja & Sin experiencia & Medio & Bajo \\
\hline P5 & M & Baja & Sin experiencia & Bajo & Bajo \\
\hline P6 & M & Baja & Sin experiencia & Bajo & Bajo \\
\hline P14 & H & Baja & Sin experiencia & Bajo & Bajo \\
\hline
\end{tabular}

Fuente: elaboración propia.

Por último, aquellos con escasas o nulas manifestaciones críticas comparten también su bajo nivel de experiencia docente, así como de dedicación y comprensión.

Lo anterior indica la probabilidad de que el experto, dada su estructura de conocimiento compleja y de multinivel (Marcelo, 2008) construya una visión crítica de su práctica y su entorno, siempre y cuando su reflexión se nutra de conocimiento conceptual y empírico sobre el fenómeno educativo como fenómeno social, más allá de su propia experiencia y sentido común. En este sentido, es preciso considerar el conocimiento académico como poderoso instrumento o herramienta conceptual que permite reconstruir las interpretaciones de las situaciones y las decisiones de intervención en la práctica, cuando se integran significativamente (Pérez Gómez, 1993).

En contraste, se puede inferir que, aun cuando se reconozcan procesos de reflexión en los profesores principiantes o sin experiencia, les es difícil interpretar críticamente una realidad que no han vivido o que no lo han hecho con suficiencia desde el otro lado del proceso educativo. Sí alcanzaron a percibir el carácter social de la educación, y en ese sentido se promovió un cambio de pensamiento; pero en tanto no estaban inmersos en una realidad donde aplicaran de manera práctica las teorías y conocimientos académicos estudiados con cierto nivel de profundidad, no se alcanzaron a visualizar con claridad los problemas sociales en la educación. Es notorio además, que la mayoría de los que tuvieron bajo nivel de estudio y por tanto, menor comprensión, no tenían práctica docente, por lo que, se infiere, tenían poco interés en la temática.

Por último, cabe mencionar que el descubrir las relaciones subyacentes de los procesos educativos se generó cierto sentimiento de incertidumbre y frustración en cuanto a qué hacer frente a las determinaciones sociales, como ocurre con los estudiantes de primer ingreso de la carrera de sociología; pero la misma reflexión llevó a considerar ideas esperanzadoras, a partir del reconocimiento de la lógica en la que se inscribe la acción docente. De ahí que uno de los participantes escribiera: 
"Queda la esperanza, de que, con su acción, el docente posibilite un cambio en los sujetos a su cargo, generando modos, formas y expresiones distintas de hacer pedagogía». (P9).

\section{Conclusiones}

El estudio del fenómeno educativo desde la sociología es vital para situarlo socialmente, pero la construcción de un pensamiento crítico puede ser más factible cuando se vincula con la práctica docente, para darle sentido a una reflexión que cuestiona lo vivido. Así entonces, la experiencia docente conjugada con el análisis profundo y comprensión de las aportaciones de las teorías de la sociología de la educación contribuye a la reflexión social y crítica en el docente.

Habrá que anotar además, que no basta con un curso o unas horas de estudio y reflexión; se requiere trabajar la mente de manera permanente; fomentar programas y espacios de estudio, análisis y discusión colectiva, donde estén presentes estas perspectivas. Insistir que la reflexión docente no implica solo atender la práctica en su ámbito pedagógico o psicológico, sino también desde lo sociológico.

El estudio de las teorías sociológicas que abordan el fenómeno de la educación, tanto desde una visión integradora como desde una postura crítica, pueden contribuir al desarrollo de un pensamiento comprometido con los procesos de enseñanza y de aprendizaje ya no como asuntos de interés individual, sino en beneficio del colectivo, de la sociedad en general. No obstante, el análisis crítico necesario puede permitir entender su importancia y empoderar su sentido para la formulación de políticas públicas que realmente busquen un cambio social.

El docente como agente intelectual y social tiene la posibilidad de tener una participación activa en la formación de actores sociales que busquen el desarrollo de sociedades justas y humanizadas.

\section{Referencias bibliográficas}

Aguilar Nery, Jesús (2003): "Aproximación a las creencias del profesorado sobre el papel de la educación formal, la escuela y el trabajo docente”. Región y Sociedad, 15 (26), 73-102.

Armenta Beltrán, Margarita; Ibarra Aguirre, Enrique y Jacobo García, Héctor M. (2015): “Transiciones y rupturas profesionales a contextos de alta clausura sociocultural". Revista Electrónica de Investigación Educativa, 17 (2), 116-129.

Baildon, Mark C. y Sim, Jasmine B. Y. (2009): "Notions of criticality: Singaporean Teachers Perspectives of Critical Thinking in Social Studies". Cambridge Journal of Education, 39 (4), 407-422.

Bourdieu, Pierre y Passeron, Jean-Claude (1996). La reproducción. Elementos para una teoría del sistema de enseñanza. México: Fontamara.

Corbally, John E. (1942). "Educational Sociology for Teachers”. The Phi Delta Kappan, 24 (7), 281-283.

Daine, Patricia y Foster, Louis (1976): "The Role of Sociology in Teacher Education”. Australian Journal of Education, 20 (3), 292-305. https://doi.org/10.1177/000494417602000306

DeCesare, Michael (2003): "The Impact of Sociological Orientation on Pedagogy: A Reconsideration of Teacher Training in Sociology Ph.D. Departments”. The American Sociologist, 34 (3), 73-96.

Dewey, John (1989). Cómo pensamos. Barcelona: Paidós.

Durkheim, Émile (1975). Educación y sociología. Barcelona: Península. 
Fernandes dos Santos, José Manuel (2008): "Valores e deontologia docente. Um estudio empírico". Revista Iberoamericana de Educación, 47 (2), 1-14, https:/ / rieoei.org/RIE/article/view/2368/3372

García Pérez, Francisco F. (2006). “¿Qué retos le plantean al profesor las nuevas realidades sociales que nos envuelven? ¿Qué perfil de profesor se considera necesario para una escuela pública, democrática e inclusiva?” en Jesús Romero Morantes, (et al.): “La formación del profesorado y la construcción social de la docencia", Con-ciencia social, (10), 40-45.

Giroux, H. (2004). Teoría y resistencia en educación. México: Siglo XXI-UNAM.

Gómez Torres, Francisco Hernando y Niño Gutiérrez, Elizabeth Magaly (2015). "La identidad profesional del profesor en educación secundaria. El posicionamiento político en la función del maestro". Revista Educación y Ciudad. Julio-diciembre (29), 187-198.

Guerrero Serón, Antonio (2007). "La doble contribución de la sociología a la formación del profesorado”. Revista Internacional de Sociología, 65 (48), 203-220. http:/ / dx.doi.org/10.3989/ris.2007.i48.74

Habermas, Jürgen (1995). Conocimiento e interés. Valencia: Universitát de Valencia.

Jacobo García, Héctor Manuel (1997): La exploración del pensamiento pedagógico de los profesores de educación básica: una contrastación con los pedagogos clásicos del primer tercio del presente siglo. Tesis doctoral. Madrid: Universidad Autónoma de Madrid.

Jackson, Philip (1998). La vida en las aulas. Madrid: Morata.

Jiménez, Felipe y Fardella, Carla (2015). "Diversidad y rol de la escuela: discursos del profesorado en contextos educativos multiculturales en clave migratoria". Revista Mexicana de Investigación Educativa, 20 (65), 419-441.

Kemmis, Stephen (2007). "Action research as a practice-changing practice". Spanish Collaborative Action Research Network (CARN) Conference, University of Valladolid, October 18-20. https://www.infor.uva. es/ amartine/MASUP/Kemmis_2007.pdf

Krichesky, Gabriela J.; Martínez Garrido, Cyntha; Martínez Peiret, Ana M.; García Barrera, Alba; Castro Zapata, Adriana y González Bustamante, Anita (2011): "Hacia un programa de formación docente para la justicia social”. REICE, 9 (4), 64-77. http://hdl.handle.net/10486/660855

Loubet Orozco, Roxana y Jacobo García, Héctor Manuel (2013): "La reflexión sociofuncional y crítica de los profesores de programas de educación para la población infantil migrante”. Revista Internacional de Educación para la Justicia Social, 2 (1), 195-212. http:/ /www.rinace.net/riejs/numeros/vol2-num1/art10.pdf.

Macotela Flores, Silvia; Flores Macías, Rosa del C. y Seda Santana, Ileana (2001): "Las creencias de los docentes mexicanos sobre el papel de la escuela y del maestro". Revista Iberoamericana de Educación, De los lectores, http://www.rieoei.org/deloslectores/106Macotela.PDF

Marcelo, Carlos (Coord.) (2008). El profesorado principiante. Inserción a la docencia. Octaedro: Barcelona.

McNamara, D. (1972): "Sociology of Education and the Education of Teachers". British Journal of Educational Studies, 20 (2), 137-147. 
Méndez Núñez, Ángel y Murillo, Javier (2017). "Reivindicando la labor intelectual y transformativa del profesorado en una Educación para la Justicia Social”. Revista Internacional de Educación para la Justicia Social, 6(1), 383-399.

Montané López, Alejandra, Aneas Alvarez, M. Asunción y Olivé Ferrer, M. Carmen (2009): “El fracaso escolar y los supuestos esenciales de la reforma: la percepción del profesorado de la educación secundaria en Cataluña (España)". Revista Iberoamericana de Educación, 49 (8), 1-13.

Murillo Torrecilla, F. Javier y Hernández Castilla, Reyes (2011): "Hacia un concepto de justicia social”. REICE, 9 (4), 8-23.

Pereyra, Diego y Pontremoli, Claudia (2014): “¿La Sociología está Pasada de Moda? Una discusión sobre la enseñanza de sociología en la escuela media en Argentina: docentes, estudiantes y propuesta curricular". Educaçao \& Realidade, 39 (1), 139-159.

Pérez Gómez, Ángel I. (1993): "La formación del docente como intelectual comprometido. La tarea de provocar la reconstrucción del conocimiento". Signos, 8 (9), 42-53.

Pérez Puente, Julio R. (2010): "El seminario alemán una estrategia pedagógica para el estudiante”. Cultura, Educación, Sociedad, 1 (1), 107-112.

Reavis, Williams C. (1928): “The Place of Educational Sociology in the Training of the Secondary-School Principal”. The Journal of Educational Sociology, 1 (6), 322-329.

Sáenz del Castillo, Andrés Á. (2009): “Formación inicial del profesorado. Propuestas”. Ágora para EF y el Deporte, 11, 7-24.

Schön, D. (1992). La formación de los profesionales reflexivos. México: Paidós.

SEP. (2014). Profesiograma para el bachillerato general modalidad escolarizada. México: SEP. http://www.dgb.sep. gob.mx/informacion-academica/profesiogramas/PROFESIOGRAMA-ACTUALIZACION-2016. pdf.

Tapia Romero, Eduardo y Valenzuela, Blanca (2015): "Percepción docente sobre el alumnado inmigrante en la frontera norte de México". Revista Internacional sobre Diversidad e Identidad en la Educación, 2 (2), 75-82.

Valli, Linda (1990): “Teaching as Moral Reflection: Thoughts on the Liberal Preparation of Teachers". Proceedings of the National Forum of the Association of Independent Liberal Arts Colleges for Teacher Education. Milwaukee, WI.

Van Manen, Max (1977). "Linking Ways of Knowing with Ways of Being Practical". Curriculum Inquiry, 6 (3), 205-228. http://dx.doi.org/10.1080/03626784.1977.11075533

\section{Nota biográfica}

Roxana Loubet-Orozco es socióloga, Máster en Ciencias Sociales y Doctora en Educación. Catedrática de tiempo completo de la Facultad de Ciencias Sociales de la Universidad Autónoma de Sinaloa, México. Su interés en la investigación se relaciona con la formación docente, relaciones de género y la práctica sociológica. Actualmente está desarrollando un proyecto sobre la inserción del egresado de sociología en el mercado laboral. 